\title{
Integration Strategies for the Personas technique within Agile Process
}

\author{
Patricia Losana \\ Depto. Ing. Informática, Escuela \\ Politécnica Superior \\ Universidad Autónoma de Madrid \\ Madrid, Spain \\ patricia.losana@estudiante.uam.es
}

\author{
John W. Castro* \\ Depto. Ing. Informática y Ciencias de \\ la Computación \\ Universidad de Atacama \\ Copiapó, Chile \\ john.castro@uda.cl
}

\author{
Silvia T. Acuña \\ Depto. Ing. Informática, Escuela \\ Politécnica Superior \\ Universidad Autónoma de Madrid \\ Madrid, Spain \\ silvia.acunna@uam.es
}

\begin{abstract}
Agile development processes are increasing their consideration over usability, integrating different user-centered design techniques throughout development. One such technique is Personas, which proposes the design of fictitious users with real preferences to drive application design. As applying this technique conflicts with the time constraints of agile development, Personas has been adapted over the years. Our objective is to determine the adoption level and type of integration, as well as to propose improvements to the Personas technique for agile development. A systematic mapping study was performed, retrieving 28 articles which we grouped by agile methodology type. We found some common integration issues irrespective of the type of agile process, such as the life cycle stage or the target user analysis, and also some frequent problems, mainly related to project timing and representing the Persona context. Based on these difficulties, we propose possible improvements, such as using templates for a preliminary Persona. This study analyzed the different integration strategies for the Personas technique within agile processes, evaluating the difficulties found and proposing solutions. The number of publications is increasing, which reveals a growing interest in the adoption of this technique to develop usable agile software.
\end{abstract}

Keywords-Personas; User Profiling; Human-Computer Interaction; User-Centered Design; Agile Methodology; Software Engineering; Systematic Mapping Study

\section{INTRODUCTION}

Usability is a software quality characteristic used in most of the classifications [1][2], which must be addressed throughout the entire interactive software development process [3][4]. Usability and User-Centered Design (UCD) techniques have been studied with increasing interest by development teams using agile software development processes (ASDP), as it has been shown that using only agile methods does not necessarily guarantee product usability [5]. To develop a usable software system, it is required to know how are the users to whom the system is destined [6][7]. There are several techniques within the Human-Computer Interaction ( $\mathrm{HCI}$ ) discipline that study and model the person that will use the software system, among which can be found the Personas technique [8], which has achieved promising results in software development [9]. ASDP have adopted $52 \%$ of the usability techniques related to requirements engineering. Of this group, Personas is the most used technique [10]. This user analysis technique is based on designing a user model from data obtained through interviews with real users, guiding the application design with the users' preferences and avoiding that the developers create a design based on their own inclinations.

The Personas technique has been systematized at the same level as the software engineering (SE) techniques through the works of [11] and [12]. Later, in the study performed by [13], the technique has been adapted to be integrated within an agile development process and has later been evaluated through a case study by [14], which allowed to test the viability and impact of applying the Personas technique adapted within a real agile project.

The next step in this line of research corresponds to analyze the state of the art of the incorporation of the Personas technique within agile processes, in order to establish how this technique is being used within agile projects and to identify potential improvements for it. For this purpose, the research work aims to identify the different integration approaches of the Personas technique through a literature review carried out by a Systematic Mapping Study (SMS), using as a reference the guidelines described in the study of [15] and [16] due to its great representativeness within the studies of $\mathrm{HCI}$ and SE. The result of the SMS is reported in the present research work.

Paper organization. In Sec. 2, we describe the Personas Technique. In Sec. 3, we present related work. In Sec. 4, we describe the research method of the SMS. In Sec. 5 we discuss the results of the SMS. Sec. 6 presents possible threats to validity, and finally, the conclusions are presented in Sec. 7.

\section{The Personas Technique}

The Personas technique, attributed to Alan Cooper [17], is a UCD tool that seeks to conceptualize the behavior of real users within user models, with the objective of improving the usability of the design. In this way, although a persona is fictitious, the objectives it aims to cover are real, since they are synthesized from observations of final users. This allows the design and development team to empathize more easily with user preferences [18]. The following is a description of the steps that make up the Personas technique of Cooper et al. [9]:

- Step 1: Identify Behavioural Variables. In this step the different behavioral aspects observed are listed. This list is known as the set of behavioral variables.

* Corresponding Author. 
- $\quad$ Step 2: Map Interview Subjects to Behavioural Variables. Once the list of behavioral variables obtained from the subject interviews has been identified, the next step is to map each interviewed subject to each behavioral variable. These behavioral variables represent either a continuous range of behaviors or multiple discrete selections.

- Step 3: Identify Significant Behavioural Patterns. In this step, groups of subjects that fall into multiple ranges or variables are observed. A group of subjects grouping six to eight different variables could represent a significant pattern of behavior, which will form the basis of a persona.

- $\quad$ Step 4: Synthesize Characteristics and Relevant Goals. In this step, the details of each of the significant behavior patterns identified in the previous step are synthesized. This synthesis should describe the potential use environment, a typical workday, and relevant relationships with others. Brief statements describing the characteristics of the behaviors are sufficient for this synthesis.

- Step 5: Check for Completeness and Redundancy. In this step, the mappings, the characteristics of the personas and their objectives are checked to determine if there are any gaps that need to be filled. It is important to make sure that the set of personas is complete, and that each persona is significantly different from the others.

- Step 6: Expand the Description of Attributes and Behaviours. Third-person narrative is very useful for conveying the attitudes, needs, and problems of the persona to other team members. A typical description should be a synthesis of the most important details observed during the investigation that are relevant to the persona.

- $\quad$ Step 7: Designate Persona Types. Development requires a target. Typically, the most specific goal is the best. The goal is to find a single persona whose needs and objectives can be completely satisfied by a single interface without disappointing any of the other personas. This is accomplished through a process of designating persona types. There are six types of personas: (i) primary, (ii) secondary, (iii) supplemental, (iv) customer, (v) served, and (vi) negative.

It is important to mention that the application of the Personas Technique impacts the use cases, since a persona model is attached to each actor. The annotated use case diagrams and the use case specification provide project stakeholders with a model providing a common understanding for deciding what the software system should do according to each persona.

\section{RELATED WORK}

Even though the Personas technique belongs to the HCI branch and not to the agile methodology, Personas has been sought to be used in agile processes to help development teams to have a better design [19]. However, the original idea of Personas may conflict with the agile philosophy in the process of obtaining details from end users regarding the system [20].

Although there are different agile methodologies, all of them are characterized by being iterative, promoting developer-client collaboration, and receiving feedback from the client throughout the development life cycle. The most relevant methodologies are: Dynamic Systems Development (DSDM) [21], Functionality Driven Development (FDD) [22], Lean Software Development [23], Scrum [24] and eXtreme Programming (XP) [25]. The agile philosophy is characterized by evaluating the functionality of prototypes with users over short iterations, which tends to have a direct impact on the usability of the design. Therefore, in order to develop usable software and prevent disuse, the integration of UCD techniques within agile methodologies has increased in the recent years [26][27]. Within these integrations, an agile version of the Personas technique stands out, which consists of a partial application of the technique at the beginning of the development and its refinement and completeness throughout the iterations. This agilized version of the Personas technique helps to overcome the time constraints that exist in the agile development process [28][29].

Different examples have been found in the agile literature proving that the Personas technique helps both to improve the usability of interfaces and to meet user requirements during the agile lifecycle. This makes the technique useful for mediating communication between developers and designers, measuring design effectiveness, and determining how a product should behave [30][31][32]. Therefore, the next step would be to know how such technique is being integrated in the different types of agile development processes in order to achieve an effective usability result in the software product, and to learn from the experiences of the different researches to unify the integration guidelines, thus improving its application in future work.

\section{RESEARCH METHOD}

The information extracted from the primary studies aims to answer the following research questions: (RQ1) What is the state of the art of the Personas technique adoption in agile processes? (RQ2) What are the main ways of integrating the Personas technique in agile software development according to the primary studies? (RQ3) What are the main difficulties of integrating the Personas technique in agile software development and what improvements can be made?

\section{A. Define the Search Strategy}

The SMS begins with the identification of the keywords, which are those that appear most frequently in the Control Group (CG) articles: a reduced set of 13 papers directly related to the research area: [19][20][26]-[31][33]-[37]. Thus, to assess the validity of the search strings formed, we checked the number of CG articles retrieved within the Scopus database, since, being the largest database, it is where the highest number of $\mathrm{CG}$ articles are most likely to be found. To obtain the keywords, a table was generated with the frequency of all the words and combinations of words that appear in the CG articles, with the help of the Atlas.ti software. Only those words directly related to the research questions and that were present in a significant percentage of the GC articles were selected.

Once the keywords were identified, several search strings were constructed. For their construction, the words were grouped into synonyms of different components: words related to (i) the Personas technique, (ii) usability, and (iii) agile processes. The logical operator AND was used to join each of 
these components, while the logical operator OR was used to include synonyms of words from the same component. The different strings generated were tested within the Scopus database, and finally we selected the one that retrieved the bigger amount of CG articles: Personas AND (usability OR user OR ucd OR "user-centered design" OR ux OR "user experience" OR hci OR "interface design" OR "interaction design") AND (agile OR "agile development" OR "extreme programming" OR scrum OR sprint $O R$ "user stories" $O R$ "agile method" $O R$ "agile software development" OR "agile process"). The criteria used to select the primary studies are summarized below.

a) Inclusion criteria: the paper is directly related to the use of the Personas technique in agile software development OR the paper describes the application of the Personas technique in agile software development OR the paper integrates the Personas technique in agile software development.

b) Exclusion criteria: the paper is a secondary study OR the paper is a primary study, but the topic is not directly related to integration or the use of the Personas technique in agile software development OR the paper is not written in English.

\section{B. Select the Studies}

Although the search string tests were performed in Scopus, the largest database of peer-reviewed literature [38], the searches were also performed in ACM Digital Library and IEEE Xplorer in order to cover more results. The databases were analyzed sequentially: first Scopus ("Title OR Abstract OR Keywords"), then ACM ("Abstract OR Title") and finally IEEE Xplorer ("Abstract OR Title"). If a duplicate appeared, the first result was kept.

A total of 104 papers were found in the different databases, among which $9 \mathrm{GC}$ articles were retrieved. After excluding the articles that had appeared duplicated, the number was reduced to 78. Next, a peer review was carried out on these articles, applying the selection criteria to the title and abstract. The selected articles were validated upon a consensus meeting, analyzing together the abstracts of the conflicting articles, thus reducing the total to 38 pre-selected articles. After the meeting, the selection criteria were again applied to the remaining articles, but this time to the complete text, obtaining a final selection of 28 primary studies [27]-[30][34][35][39]-[60].

\section{RESUlts AND Discussion}

\section{A. State of the Art of the Personas technique adoption}

To assess the state of the art of the adoption of the Personas technique in agile processes, each of the 28 selected studies was classified according to the type of agile process on which it was integrated. Fig. 1 synthesizes the results using two bubble scatter plots. The upper graph represents the number of articles published per year according to the type of publication (conference, journal or book chapter). Similarly, the lower graph plots the type of publication against the agile methodology on which it has been integrated. Thus, the bubbles are located at the intersections between the two axes and their size is proportional to the number of publications for each combination of values.
Although there have been studies integrating the Personas technique in agile processes since 2003 , the interest in its integration in agile developments is increasing since 2016. This increase in the application of the technique may be due to the increasing use of agile methodologies because of their benefits. As the interest grows, the need to know the users arises, and therefore the use of the Personas technique is increasing. In addition, most primary studies have focused on Scrum and XP agile processes, and have been published in specialized conferences and journals, suggesting that the interest of the scientific community in integrating this technique in agile processes is increasing.

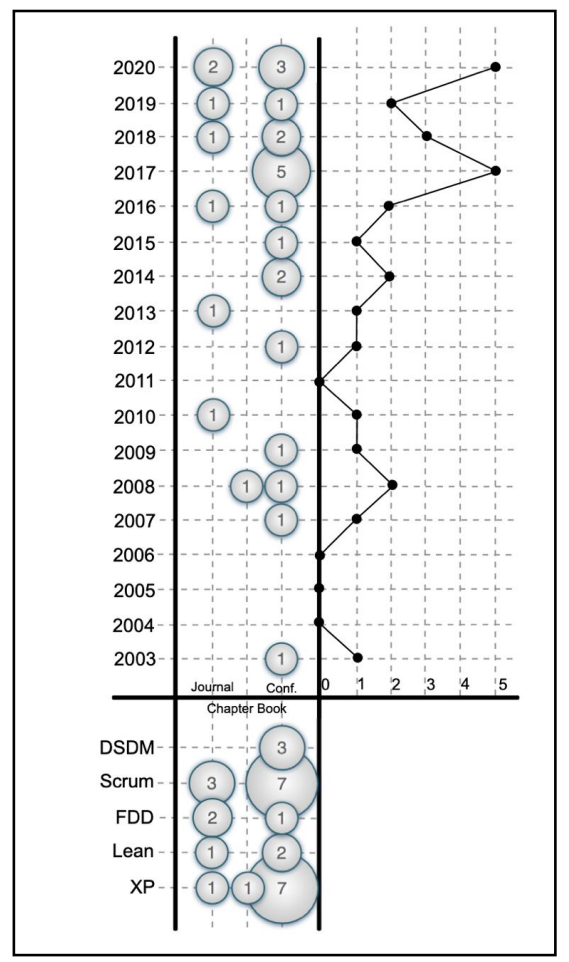

Figure 1. Mapping for the primary study distribution.

\section{B. Main ways of integrating the Personas technique}

We identified and extracted the main forms of integration of the Personas technique in agile software development for the selected articles, the description of how this integration was carried out and the life cycle activity where it was integrated. A synthesis of these results is shown on a mind map in Fig. 2.

1) DSDM: The studies conducted on DSDM agile processes create the personas models from both an interview and an analysis of the user stories. In the case of [39], instead of a narrative, they create drawn sketches of the personas based on the information obtained within the interviews. In [40], a previous design thinking session is carried out in which user stories are analyzed among all team members. In both cases the technique is integrated during the elucidation and requirements analysis activity, and additionally in [41] it is also integrated during the planning and design activity. In all three studies they validate the assignment of personas to user stories with the end users before starting the design. Moreover, in all of them, they validate each of the solutions after elaborating their designs. 


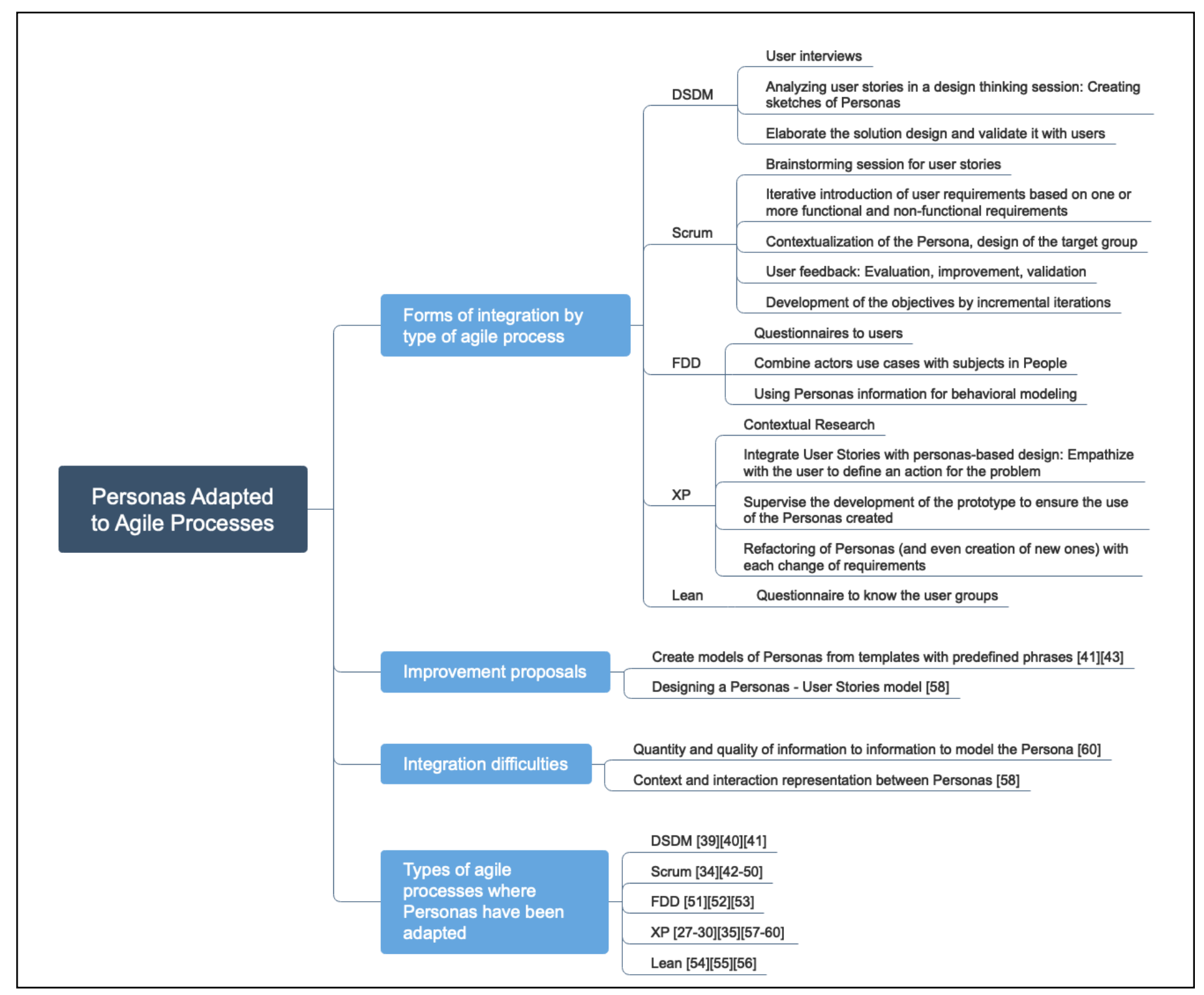

Figure 2. Main forms of integrations of the Personas technique in agile software development, main difficulties and proposed improvements.

2) Scrum: The studies that have integrated Personas into Scrum propose creative team sessions prior to the start of development to complete the Personas narratives. There are several studies in which they conduct a brainstorming session with students [42][43][44], where they complete the Personas narratives with previously generated sentences, and later associate them to the most convenient User Stories [45]. In the study by [46], it is proposed to use mind maps to connect the different Personas. The studies [34][47][48] associate each persona with a specific context, with a short description with preferences and a motivation that makes it easier for developers to empathize with end users during development. They all address user goals in incremental iterations, validating the functionality of the goals with users after each iteration. Moreover, in [49][50] they include non-functional requirements as goals as well, in order to obtain high-fidelity prototypes.
3) FDD: As for studies that have integrated Personas in FDD, they seek to analyze the interaction of people to establish behavioral patterns. In the studies of [51] and [52], they abstract patterns from user stories and assign them to specific subjects, and in [53] they further conduct interviews involving emotional analysis experts in order to more easily identify end-user personalities.

4) Lean: The integration of Personas in the studies analyzed on Lean start by knowing the user groups targeted by the development, either through questionnaires [54] or contextual investigations [55]. In [56] they group the results into clusters of users based on the preferences and behaviors found, customizing subsequent designs for the patterns found in each cluster.

5) XP: Finally, the studies carried out on the integration of Personas in XP interview and investigate the context of users in 
order to empathize more easily with them, and thus orient the development to their preferences [32][33][34][39][57][58][59]. Furthermore, [27] and [30] propose to collect information asynchronously to the project development as the team receives new information from users, refactoring and even creating new personas if they fit better the new user requirements. As in [46], in the study by [60] it is also proposed to design a mind map to connect what the persona wants and how they want it, using colors to highlight what is most relevant.

\section{Main difficulties of integrating the Personas technique}

Throughout the literature review, two main difficulties have been found to integrate the Personas technique in agile software development, generalized throughout the different studies. The main difficulty is to find the amount of necessary and sufficient information that should appear in the initial description of the persona. It should be detailed enough for the development team to empathize with the user's needs, but not as detailed as to conflict with the time restrictions within an agile process [41] [60]. An interesting solution could be to create the initial models of personas from templates with predefined phrases, as was proposed in [41] and [43]. Although the personas created by selfreported information during interviews may not be reliable [60], after the analysis performed over all the primary studies, we consider that this could be standardized within the Personas technique integrated to agile methodologies. This way, the first persona model would be created with a much lower temporal impact over the project. The first persona sketch would be simple, but it would be refined over iterations, as applied in studies [28][52][51][55][59].

The other difficulty shared by the different studies is to represent the context where a persona wants to perform an action, and the possible interaction with other personas within one same requirement [58]. Personas are created independently of each other, with the purpose of solving specific use cases [39] [41][47]. In the study of [58] they propose to design an EntityRelationship model to allow differentiating the relationships between the different personas and their user stories.

Within the model there would be three entities: User Story, Persona and Navigation Relationship. The User Story entity would have a user value attribute, with the objective of prioritizing the list of requirements. On the other hand, the Persona entity would contain the attributes related to context of use, so that it would be possible to differentiate between different types of requirements according to the user. Finally, the Navigation Relationship entity would include attributes representing the interactions between Persona and User Stories, thus allowing different contexts of use between different Personas for the same User Story and, therefore, representation of more complex usage scenarios.

\section{VALIDITY THREADS}

Throughout this study, certain aspects that could jeopardize the validity of the study have been assessed. The main threat to its validity is related to the possibility of bias in the selection process of the primary studies. To reduce this bias, we followed the guidelines proposed by the authors [15][16]. In addition, this SMS was carried out using three databases (Scopus, ACM, and
IEEE Xplore), since they were considered the most relevant for the purpose of this search. However, if additional databases had been included, new results and complementary information would have been obtained. Numerous tests were also carried out to ensure the adequacy of the search string, checking that the maximum possible number of papers belonging to the $\mathrm{CG}$ were returned by the final search string. Another threat to validity is related to the application of the selection criteria and the analysis of the abstracts of the articles found. In order to minimize subjectivity, the selection process was carried out in parallel by two members of the research team, and the selected articles were subsequently agreed upon in a group meeting.

\section{CONCLUSIONS}

Throughout this work, we have presented a secondary study on the integration of the Personas technique over different types of agile processes, with the objective of understanding the current state of the art of integration and to establish a knowledge base that would allow proposing future improvements to the technique. The study started by identifying the keywords in a set of articles called Control Group. These keywords were combined to formulate a search string that allowed us to carry out an in-depth analysis of all primary studies related to the integration of both concepts (the Personas technique on agile methodologies) over different databases. Subsequently, we applied a selection criterion to exclude those publications that did not contain the information to answer the research questions. From these 28 studies analyzed in depth, it has been possible to see that the integration of the Personas technique in agile developments has been increasing since 2016, which reflects a growing interest of the scientific community in the field, especially within the agile processes Scrum and XP.

After the synthesis of the results of the different publications, we have observed that, regardless of the type of agile process in which the Personas technique was integrated, there were some common aspects among them. On the one hand, integration always takes place at least during the activity of elucidation and requirements analysis, although it may also involve other activities of the software development process. In addition, regarding the integration steps, the first step always consists of performing an analysis of the target users, either by questionnaires, interviews, or brainstorming. This step allows to obtain a first persona model that can be refined or adapted according to new user requests that come with each iteration. On the other hand, the main difficulties in integrating the technique within the agile methodology are related to the difference in paradigm between User-Centered Design (where usability and detailed knowledge of the end user is a priority), and agile development (where the objective is to cover functionalities from early iterations with value for the client, affecting the time dedicated to the design).

In conclusion, this secondary study has analyzed numerous articles focused on the integration of the Personas technique within agile processes, in which different integration approaches, difficulties and proposed solutions have been found. This comprehensive analysis of articles from reliable sources provides support for the development and application of this technology on the future, aiming to obtain agile development processes with increasingly user-centered results. Future work 
will attempt to propose a more agile technique to validate it in a case study.

\section{ACKNOWLEDGMENT}

This research was funded by Spanish Ministry of Science, Innovation and Universities research grant PGC2018-097265B-I00 and MASSIVE project (RTI2018-095255-B- I00). This research was also supported by the Madrid Region R\&D programme (project FORTE, P2018/TCS-4314).

\section{REFERENCES}

[1] ISO/IEC Std. 25010. (2011). Systems and Software Engineering Systems and Software Quality Requirements and Evaluation (SQuaRE) System and Software Quality Models. ISO/IEC.

[2] ISO Std. 9241-11. (2018). Ergonomics of Human-System Interaction Part 11: Usability: Definitions and Concepts. ISO.

[3] Shneiderman, B., Plaisant, C., Cohen, M., Jacobs, S., Elmqvist, N., and Diakopoulos, N. (2016). Designing the User Interface: Strategies for Effective Human-Computer Interaction. Pearson.

[4] Ferreira, J. M., Acuña, S. T., Dieste, O., Vegas, S., Santos, A., Rodríguez, F., and Juristo, N. (2020). Impact of usability mechanisms: An experiment on efficiency, effectiveness and user satisfaction. Information and Software Technology, 117, 1-17.

[5] Fox, D., Sillito, J., \& Maurer, F. (2008). Agile methods and user-centered design: How these two methodologies are being successfully integrated in industry. In Proceedings of the Agile 2008 Conference. Toronto, ON, Canada, pp. 63-72.

[6] Nielsen, J., Berger, J. M., Gilutz, S., and Whitenton, K. (2003). Return on Investment (ROI) for Usability, 4th Edition, Nielsen Norman Group. [Online] https://n9.cl/9cwd. Last Access on December, 2020

[7] Rodríguez, F. D., Acuña, S. T., and Juristo, N. (2015). Design and programming patterns for implementing usability functionalities in web applications. Journal of Systems and Software, 105, 107-124.

[8] Cooper, A., Reimann, R., and Cronin, D. (2007). About Face 3.0: The Essentials of Interaction Design. Wiley Publishing, Indianápolis: Wiley Publishing.

[9] Cooper, A., Reimann, R., Cronin, D. (2007). About Face 3.0: The Essentials of Interaction Design. Wiley Publishing, Indianápolis.

[10] Magües, D. A., Castro, J., \& Acuña, S. T. (2016). Requirements engineering related usability techniques adopted in agile development processes. In Proceedings of the Twenty-Eighth International Conference on Software Engineering and Knowledge Engineering (SEKE'16). San Francisco Bay, California, USA, pp. 537-542.

[11] Castro, J. W. (2009). Extensión de la Técnica Personas para su Incorporación en la Actividad de Análisis del Proceso de Ingeniería del Software. Trabajo de Fin de Máster. Directora: Acuña, S.T. Universidad Autónoma de Madrid.

[12] Acuña, S. T., Castro, J. W., Juristo, N. (2012). A HCI Technique for Improving Requirements Elicitation. Information and Software Technology, 54(12): 1357-1375.

[13] Magües, D. A. (2016). Transformación de Técnicas de Usabilidad relacionadas con las Actividades de la Ingeniería de Requisitos para su Incorporación en los Procesos de Desarrollo Ágil. Trabajo Fin de Máster, Escuela Politécnica Superior de Informática, Universidad Autónoma de Madrid. Directores: Acuña, S. T., y Castro, J. W.

[14] Losana, P. (2019). Aplicación de la Técnica Personas en un Proyecto de Desarrollo Ágil con Scrum. Trabajo Fin de Grado, Escuela Politécnica Superior de Informática, Universidad Autónoma de Madrid. Directora: Acuña, S. T.

[15] Kitchenham, B., and Charters, S. (2007). Guidelines for Performing Systematic Literature Reviews in Software Engineering. Tech. rep., Keele University and Department of Computer Science University of Durham.

[16] Kitchenham, B. A., Budgen, D., \& Brereton, O. P. (2011). Using mapping studies as the basis for further research-a participant-observer case study. Information and Software Technology, 53(6): 638-651.
[17] Cooper, A. (2004). The Inmates are Running the Asylum: Why High-Tech Products Drive Us Crazy and How to Restore the Sanity. Sams - Pearson Education, Indianápolis.

[18] Caballero, L., Moreno, A. M., \& Seffah, A. (2014, September). Persona as a tool to involving human in agile methods: Contributions from HCI and marketing. In Proceedings of the Intern. Conf. on Human-Centred Software Engineering (HCSE'14). Paderborn, Germany, pp. 283-290.

[19] Anwar, S., Motla, Y. H., Siddiq, Y., Asghar, S., Hassan, M. S., \& Khan, Z. I. (2014, December). User-centered design practices in scrum development process: A distinctive advantage?. In Proceedings of the 17th IEEE Intern. Multi Topic Conference 2014. Karachi, Pakistan, pp. 161-166.

[20] Chamberlain, S., Sharp, H., \& Maiden, N. (2006). Towards a Framework for Integrating Agile Development and User-centred Design. In: Abrahamsson, P., Marchesi, M., and Succi, G. (eds.) Extreme Programming and Agile Processes in Soft. Eng., pp. 143-153.

[21] Stapleton, J. (Ed.). (2003). DSDM: Business Focused Development. Pearson Education.

[22] Palmer, S. R., and Felsing, M. (2001). A Practical Guide to FeatureDriven Development. Prentice Hall.

[23] Poppendieck, M., and Poppendieck, T. (2003). Lean Software Development: An Agile Toolkit. Addison-Wesley Professional.

[24] Schwaber, K., and Beedle, M. (2002). Agile Software Development with Scrum, 1st Edition. Pearson.

[25] Beck, K., \& Gamma, E. (2000). Extreme programming explained: embrace change. Addison-Wesley Professional.

[26] Sy, D. (2007). Adapting usability investigations for agile user-centered design. Journal of Usability Studies, 2(3): 112-132.

[27] Wolkerstorfer, P., Tscheligi, M., Sefelin, R., Milchrahm, H., Hussain, Z., Lechner, M., and Shahzad, S. (2008). Probing an agile usability process. In Proceedings of the CHI Conference on Human Factors in Computing Systems (CHI'08). Florence, Italy, pp. 2151-2158.

[28] Haikara, J. (2007). Usability in agile software development: extending the interaction design process with personas approach. In Proceedings of the International Conference on Extreme Programming and Agile Processes in Software Engineering (XP'07). Como, Italy, pp. 153-156.

[29] Rahim, W. A., Isa, W. M., Lokman, A. M., Taharim, N. F., and Wahid, N. D. (2014). Engineering m-learning using agile user-centered design. In Proceedings of the 2014 Eighth Intern. Conf. on Next Generation Mobile Apps, Services and Technologies. Oxford, UK, pp. 60-65.

[30] Broschinsky, D., \& Baker, L. (2008). Using persona with XP at LANDesk Software, an Avocent company. In Proceedings of the Agile 2008 Conference. Toronto, ON, Canada, pp. 543-548.

[31] Hussain, Z., Slany, W., and Holzinger, A. (2009b). Current state of agile user-centered design: A survey. In Proceedings of the Symposium of the Austrian HCI and Usability Engineering Group (USAB'09). Linz, Austria, pp. 416-427.

[32] Øvad, T., and Larsen, L. B. (2015). The prevalence of UX design in agile development processes in industry. In Proceedings of the 2015 Agile Conference. Washington, DC, USA, pp. 40-49.

[33] Hudson, W. (2003). Adopting user-centered design within an agile process: A conversation. Cutter IT Journal, 16(10): 5-12.

[34] Hudson, W. (2013). User stories don't help users: Introducing Persona stories. Interactions, 20(6): 50-53.

[35] Hussain, Z., Milchrahm, H., Shahzad, S., Slany, W., Tscheligi, M., and Wolkerstorfer, P. (2009). Integration of eXtreme Programming and usercentered design: Lessons learned. In Proceedings of the Intern. Conf. on Agile Processes and Extreme Programming in Software Engineering (XP'09). Sardinia, Italy, pp. 174-179.

[36] Najafi, M., and Toyoshiba, L. (2008). Two case studies of user experience design and agile development. In Proceedings of the Agile 2008 Conference. Toronto, ON, Canada, pp. 531-536.

[37] Nakao, Y., Moriguchi, M., and Noda, H. (2014). Using agile software development methods to support human-centered design. NEC Technical Journal, 8(3): 37-40.

[38] Scopus. An Eye on Global Research: 5,000 Publishers. Over $71 \mathrm{M}$ Records and 23,700 Titles, Amsterdam, The Netherlands, Elsevier, 2018. 
[Online].

Available:

https://www.elsevier.com/ data/assets/pdf_file/0017/114533/SC_FS_overview_WEB.pdf

[39] Vilchez-Sandoval, J., Vasquez-Paragulla, J., and Llulluy-Nuñez, D. (2020). On the of use agile methodologies to re-design a Networks and Data Communications course. In Proceedings of the IEEE World Conf. on Engineering Education (EDUNINE'20). Bogotá, Colombia, pp. 1-5.

[40] Parizi, R., Moreira da Silva, M., Couto, I., Trindade, K., Plautz, M., Marczak, S., Conte, T., and Candello, H. (2020). Design thinking in software requirements: What techniques to use? A proposal for a recommendation tool. In Proceedings of the 23rd Ibero-American Conf. on Software Engineering (CIbSE'20), Curitiba, Brazil, pp. 1-14.

[41] Cleland-Huang, J., Babar, M. A., \& Mirakhorli, M. (2014, May). An inverted classroom experience: engaging students in architectural thinking for agile projects. In Companion Proceedings of the 36th International Conference on Software Engineering (ICSE Companion'14). Hyderabad, India, pp. 364-371.

[42] Quade, S., and Schlüter, O. (2020). Ideation: Generating as many ideas as possible. Logos, 31(1): 48-53.

[43] Gaikwad, V., Joeg, P., and Joshi, S. (2017). AgileRE: Agile Requirements management tool. In Proceedings of the Computational Methods in Systems and Software (CoMeSySo'17). Szczecin, Poland, pp. 236-249.

[44] Hakim, J., Spitzer, T., and Armitage, J. (2003). Sprint: Agile specifications in shockwave and flash. In Proceedings of the 2003 Conference on Designing for User Experiences (DUX'03). San Francisco, CA, USA, pp. 1-14.

[45] Choma, J., Zaina, L. A., \& Beraldo, D. (2015, August). Communication of design decisions and usability issues: a protocol based on Personas and Nielsen's heuristics. In International Conference on Human-Computer Interaction, pp. 163-174

[46] Sekar, B. (2017). Enterprise software experience design: Journey and lessons. In Proceedings of the IFIP Conference on Human-Computer Interaction (INTERACT'17). Bombay, India, pp. 356-359.

[47] Dirks, S. (2020). Persona design in participatory agile software development. In Proceedings of the 22nd Intern. Conference on HumanComputer Interaction (HCI'20). Copenhagen. Denmark, pp. 52-64.

[48] Choma, J., Zaina, L. A., \& Beraldo, D. (2016, July). UserX story: incorporating UX aspects into user stories elaboration. In Intern. Conf. on Human-Computer Interaction, pp. 131-140.

[49] Paiva, S. C., and Carvalho, D. B. F. (2018). Software creation workshop: A capstone course for business-oriented software engineering teaching. In Proceedings of the XXXII Brazilian Symposium on Software Engineering (SBES'18). Sao Carlos, Brazil, pp. 280-288.

[50] Losada, B. (2018). Flexible requirement development through user objectives in an agile-UCD hybrid approach. In Proceedings of the XIX
International Conference on Human Computer Interaction (Interacción'18). Palma, Spain, pp. 1-8.

[51] Forbrig, P., and Dittmar, A. (2019). Applying agile methods and personas to S-BPM. In Proceedings of the 11th International Conference on Subject-Oriented Business Process Management (S-BPM ONE'19). Sevilla, Spain, pp. 1-10.

[52] de Carvalho, C. R. M. (2010). MEX experience boards: A set of agile tools for user experience design. In Proceedings of the IX Symposium on Human Factors in Computing Systems (IHC'10). Belo Horizonte Minas Gerais, Brazil, pp. 213-216.

[53] Abdullah, N. N. B., Grundy, J., McIntosh, J., How, Y. C., Saharuddin, S., Tat, K. K., Ye, E. S., Rastom, A. J. A., \& Othman, N. L. (2020, August). Using Work System Design, User Stories and Emotional Goal Modeling for an mHealth System. In 2020 IEEE First Intern. Work. on Requirements Engineering for Well-Being, Aging, and Health (REWBAH). Zurich, Switzerland, pp. 1-10.

[54] Perdana, R. A., Suzianti, A., and Ardi, R. (2017). Crowdfunding website design with lean product process framework. In Proceedings of the $3 r d$ International Conference on Communication and Information Processing (ICCIP'17). Tokyo, Japan, pp. 369-374.

[55] Bhattarai, R., Joyce, G., \& Dutta, S. (2016, July). Information security application design: understanding your users. In International Conference on Human Aspects of Information Security, Privacy, and Trust, pp. 103113.

[56] Mereu, S., Newman, M., Peterson, M., Taylor, E., White-Sustaita, J., and Yeats, D. (2017). Top-down vs bottom-up approaches to user segmentation: The best of both worlds. In Proceedings of the Human Factors and Ergonomics Society 2017 Annual Meeting, 61(1): 515-519.

[57] Sohaib, O., Solanki, H., Dhaliwa, N., Hussain, W., and Asif, M. (2019). Integrating design thinking into eXtreme Programming. Journal of Ambient Intelligence and Humanized Computing, 10, 2485-2492.

[58] Sedeño, J., Schön, E. M., Torrecilla, C. J., Thomaschewski, J., Escalona, M. J., and Mejías, M. (2017). Modelling agile requirements using contextbased Persona stories. In Proceedings of the 13th International Conference on Web Information Systems and Technologies (WEBIST'17), Porto, Portugal, pp. 196-203

[59] Hussain, Z., Lechner, M., Milchrahm, H., Shahzad, S., Slany, W., Umgeher, M., Vlk, T., Köffel, C., Tscheligi, M., and Wolkerstorfer, P. (2012). Practical usability in XP software development processes. In Proceedings of the Fifth International Conference on Advances in Computer Human Interactions (ACHI'12), Valencia, Spain, pp. 208-217.

[60] Khanh, N. T., Daengdej, J., and Arifin, H. H. (2017). Human stories: A new written technique in agile software requirements. In Proceedings of the 6th International Conference on Software and Computer Applications (ICSCA'17). Bangkok, Thailand, pp. 15-22. 